\title{
NOTE
}

\section{Solubilization of Progesterone and its Derivatives into Gemini Surfactant Solutions}

\author{
Hiroki Ideguchi, Yuya Takasaki, Shintaro Kondo, Mai Nakanose, Nana Teradahira, \\ Yumeka Yamamoto, Umi Mouri, and Hiromichi Nakahara* \\ Department of Industrial Pharmacy, Daiichi University of Pharmacy, 22-1 Tamagawa-cho, Minami-ku Fukuoka 815-8511, JAPAN
}

\begin{abstract}
The solubilization of poorly water-soluble progesterone derivatives into micelles of a gemini surfactant was investigated in an aqueous medium. The aqueous solubility at different temperatures was determined spectroscopically using an ultraviolet visible light spectrophotometer. Thermodynamic parameters for the solubilization were calculated under the basis of the solubility change against temperature. The solubility of progesterone was quite low and remained constant below the critical micelle concentration $(\mathrm{cmc})$ of the surfactant. On the other hand, the solubility increased considerably with increasing surfactant concentration above the cmc. It was suggested that the solubilizates were located in the vicinity of polar regions of micelles.
\end{abstract}

Key words: progesterone, gemini surfactant, solubilization, micelle

\section{Introduction}

The solubility of drugs is one of important physicochemical properties for drug delivery systems. In general, drugs with poor water solubility have a low drug absorption rate, which results in poor bioavailability. The solubility also greatly influences the ability to deliver drugs to target tissues and organs and achieve the desired drug concentration $^{1)}$.

Progesterone is an endogenous steroid sex hormone that plays a multifunctional role, especially in the female reproductive system. Its deficiency, especially in female menopause, causes various acute or chronic gynecological disorders such as headache, hot flashes, dizziness, and vaginal dryness $^{2,3)}$. Therefore, progesterone replacement therapy is performed to relieve such symptoms. Oral administration of progesterone and its derivatives entails many obstacles, such as low bioavailability due to the low water solubility $(10 \mu \mathrm{g} / \mathrm{mL})^{4)}$, short half-life due to the hepatic first-pass effect ${ }^{5}$, and enzymatic degradation in the digestive tract ${ }^{6}$.

One of the approaches for improving the water solubility of hydrophobic drugs is the use of surfactant aqueous solutions. There are a few reports on the solubilization of progesterone in typical ionic surfactant solutions, such as sodium dodecyl sulfate (SDS) and bile acids ${ }^{7-9)}$, and in nonionic surfactant solutions ${ }^{10)}$. Commonly, nonionic surfactant solutions exhibit a much lower critical micelle concentration $(\mathrm{cmc})$ and are therefore used as efficient solubilizers in various fields of industry. We have investigated the solubilization of poorly soluble substances using gemini surfactant solutions ${ }^{11,12)}$. Gemini surfactants are a dimer of typical surfactants in which the surfactant molecules are linked by a spacer. In comparison to the corresponding monomer surfactants, gemini surfactants have excellent properties, such as much smaller cmc and superior surface activity ${ }^{13,14)}$. In this regard, gemini surfactants have the unique property that the value of cmc is comparable to that of nonionic surfactants despite them having a low molecular weight and a charge in the hydrophilic group. Therefore, gemini surfactants are expected to be useful and functional solubilizers.

In the present study, $14-6-14,2 \mathrm{Br}^{-}$gemini surfactant, which exhibits the simplest chemical structure, has relative ease of synthesis among gemini surfactants, and has been examined extensively by many researchers, was used as a model solubilizer of progesterone. In addition to progesterone, we also utilized some of its derivatives, $17 \alpha$-hydroxyprogesterone and medroxyprogesterone, as solubilizates. The derivatives are used in the clinical field and have only a few structural differences from progesterone. Their use was expected to provide useful information on the relationship between structure and solubilization efficacy. Mea-

\footnotetext{
*Correspondence to: Hiromichi Nakahara, Department of Industrial Pharmacy, Daiichi University of Pharmacy, 22-1 Tamagawa-cho, Minami-ku Fukuoka 815-8511, JAPAN

E-mail: nakahara@daiichi-cps.ac.jp

Accepted January 31, 2020 (received for review January 6, 2020)

Journal of Oleo Science ISSN 1345-8957 print / ISSN 1347-3352 online

http://www.jstage.jst.go.jp/browse/jos/ http://mc.manusriptcentral.com/jjocs
} 
surement for solubilization was done in the temperature range of 288.2 to $308.2 \mathrm{~K}$ to evaluate the thermodynamic transfer of the solubilizates from the bulk to the 14-6$14,2 \mathrm{Br}^{-}$micelle. The solubilization site of progesterone and the derivatives in the micellar aggregate was investigated by employing absorption spectra.

\section{Experimental}

\subsection{Materials}

Hexanediyl-1,6-bis (dimethyltetradecylammonium bromide) (abbr. 14-6-14,2Br ${ }^{-}$) was synthesized via a previously reported procedure ${ }^{15)}$. The crude crystal obtained was purified by recrystallization three times from acetone/ ethanol mixed solvents. The identification was checked by ${ }^{1} \mathrm{H} \mathrm{NMR},{ }^{13} \mathrm{C} \mathrm{NMR}$, and elemental analysis, as mentioned in a previous paper ${ }^{16)}$. Progesterone (abbrev. PROG, >97\%) was purchased from Sigma-Aldrich (MO, USA). $17 \alpha$-hydroxyprogesterone caproate (17-OHPC, >98\%), medroxyprogesterone (MP, >98\%), and 1-octanol ( $>$ 99.5\% ) were purchased from Tokyo Chemical Industry Co., Ltd. (Tokyo, Japan). $n$-dodecane (99\%) was obtained from Nacalai Tesque (Kyoto, Japan). These reagents were used as received. The water utilized in the whole experiments was from a Direct-Q UV3 water purification system(Merck, Darmstadt, Germany) with a resistivity of $18 \mathrm{M} \Omega \cdot \mathrm{cm}$.

\subsection{Methods}

14-6-14,2Br ${ }^{-}$solutions suspended in the solubilizates, which were contained in glass vials and agitated with a rotor, were kept under thermostat controlled conditions to within $\pm 0.1 \mathrm{~K}$ at $288.2,298.2$, and $308.2 \mathrm{~K}$ for $24-48 \mathrm{~h}$ to attain complete solubilization equilibrium. Prior to the measurement, the suspensions were filtrated through a membrane filter with a pore size of $0.1 \mu \mathrm{m}$ (Millipore MILLEX VV) to remove extra undissolved materials. The solubilizate concentrations in the surfactant solutions were determined spectrophotometrically with a UV/VIS spectrophotometer (V-530, Jasco, Tokyo, Japan). The points in the figures are mean \pm S.D. The set of measurements was repeated at least three times to ensure repeatability.

\section{Results and Discussion}

\subsection{Solubility of the steroids in $14-6-14,2 \mathrm{Br}^{-}$solutions}

The chemical structures of the steroids used here are shown in Fig. 1. 17-OHPC and MP exhibit small differences in structure from PROG: a caproic acid ester at C-17 for 17-OHPC and a hydroxy at C-17 and methyl group at C-6 for MP. In 1-octanol, the steroids have significant UV absorbances at $240 \mathrm{~nm}$. Figure 2 shows plots of the absorbances against steroid concentration. The change in absorbance
(A)

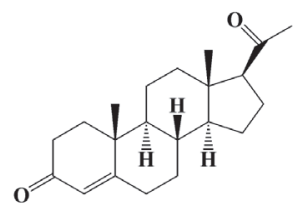

(B)

(C)

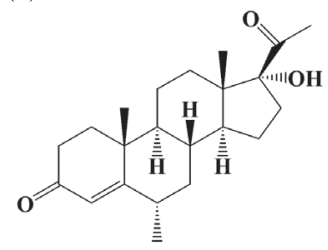

Fig. 1 Chemical structures of (A) PROG, (B) 17-OHPC, and $(\mathrm{C}) \mathrm{MP}$

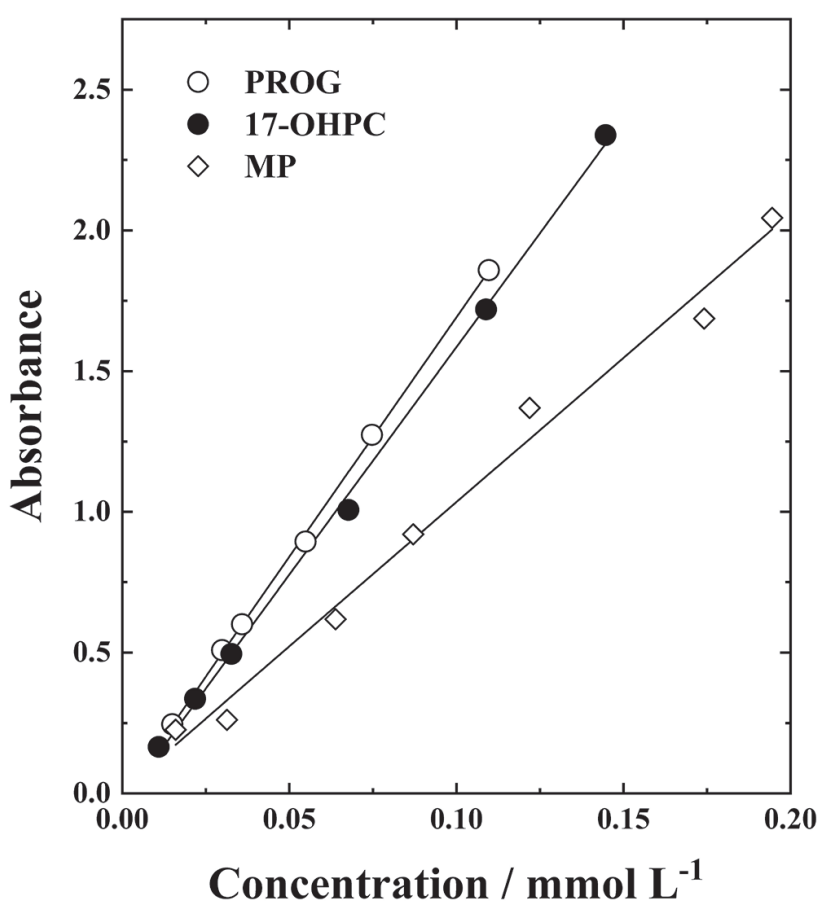

Fig. 2 Plots of absorbance of PROG, 17-OHPC, and MP in 1-octanol against concentration.

for all of the steroids indicates good linearity with respect to concentration: $r^{2}=0.999$ for PROG, 0.998 for 17-OHPC, and 0.988 for MP. Thus, the molar extinction coefficients $(\varepsilon)$ at $240 \mathrm{~nm}$, which are based on the slope in Fig. 2, are $1.69 \times 10^{4}, 1.59 \times 10^{4}$, and $1.01 \times 10^{4} \mathrm{~mol}^{-1} \mathrm{~L} \mathrm{~cm}^{-1}$ for PROG, 17-OHPC, and MP, respectively. The $\varepsilon$ value of PROG agrees well with the reference value $\left(1.56 \times 10^{4} \mathrm{~mol}^{-1}\right.$ $\left.\mathrm{L} \mathrm{cm}^{-1}\right)^{17)}$.

The solubilizate concentrations in the $14-6-14,2 \mathrm{Br}^{-}$surfactant solutions were determined by UV spectroscopy 


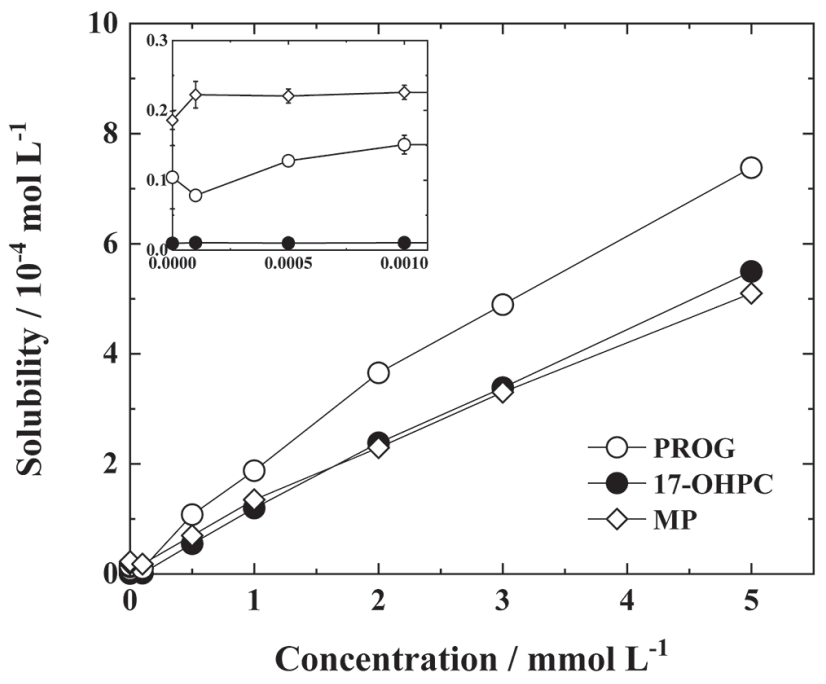

Fig. 3 Solubility changes of PROG, 17-OHPC, and MP with $14-6-14,2 \mathrm{Br}^{-}$concentration at $298.2 \mathrm{~K}$. The inset shows the enlarged view below the cmc.

using the $\varepsilon$ values. The solubilities of the steroids at 298.2 $\mathrm{K}$ are plotted against $14-6-14,2 \mathrm{Br}^{-}$concentration in Fig. 3 . The critical micelle concentration (cmc) of $14-6-14,2 \mathrm{Br}^{-}$is $0.161 \mathrm{mmol} \mathrm{L}^{-1}$ at $298.2 \mathrm{~K}^{16)}$. The solubilizate concentrations below the $\mathrm{cmc}$ remain almost constant (see the inset in Fig. 3). The solubility in water at $298.2 \mathrm{~K}$ of PROG, 17OHPC, and MP was found to be 0.0104, 0.000988, and $0.0186 \mathrm{mmol} \mathrm{L}^{-1}$, respectively. These values support the poor solubility of these compounds in water and imply their strength of hydrophobicity: 17-OHPC $>$ PROG $>$ MP. An increment of surfactant concentration above the cmc considerably increases the steroid solubility. Furthermore, the solubility varies linearly with the surfactant concentration. The difference in solubility change from below to above the cmc is attributed to the transfer of the solubilizates from the aqueous bulk to the interior of micelles ${ }^{18,19)}$. The solubility of PROG in $5 \mathrm{mmol} \mathrm{L}^{-1}$ surfactant solution is the highest among the steroids used here. This suggests that important factors for the solubilization to micelles include not only the hydrophobicity of the solubilizates but also the van der Waals interaction and hydrogen bonding between the steroids and the surfactants constituting micelles. The solubility plots at the different temperatures of 288.2 and $308.2 \mathrm{~K}$ are shown in Figs. S1-3 in the Supplementary Material.

\subsection{Thermodynamic parameters for micellar solubilization}

The mole fractions of the solubilizates in the aqueous phase $\left(X_{\mathrm{R}}^{\mathrm{W}}\right)$ and the aggregate phase $\left(X_{\mathrm{R}}^{\mathrm{A}}\right)$ are given by Eqs. 1 and 2, respectively:

$$
X_{\mathrm{R}}^{\mathrm{W}}=\frac{[\mathrm{R}]}{55.5+[\mathrm{R}]+\mathrm{cmc}}
$$

Table 1 Thermodynamic parameters (in $\mathrm{kJ} \mathrm{mol}^{-1}$ ) of the steroids for the transfer from the aqueous bulk to the surfactant micelle at $298.2 \mathrm{~K}$.

\begin{tabular}{cccc}
\hline steroids & $\Delta G^{0}$ & $\Delta H^{0}$ & $-T \Delta S^{0}$ \\
\hline PROG & -32.0 & -13.8 & -18.3 \\
17-OHPC & -38.7 & 10.6 & -49.3 \\
MP & -31.2 & -4.7 & -26.4 \\
\hline
\end{tabular}

$$
X_{\mathrm{R}}^{\mathrm{A}}=\frac{[\mathrm{R}]_{\mathrm{t}}-[\mathrm{R}]}{(C-\mathrm{cmc})+\left([\mathrm{R}]_{\mathrm{t}}-[\mathrm{R}]\right)}
$$

where the subscript $\mathrm{R}$ refers to the solubilizate and the superscripts $\mathrm{W}$ and $\mathrm{A}$ refer to the aqueous phase (water) and the aggregate phase, respectively. $[R]$ is the value of solubilizate concentration (in $\mathrm{mol} / \mathrm{L}$ ) in the aqueous medium, which is obtained as the mean value of the solubilizate concentrations below the $\mathrm{cmc}$. $[\mathrm{R}]_{\mathrm{t}}$ is the total equivalent concentration of the solubilizates in the surfactant solution, and $C$ is the total surfactant concentration. The chemical potential $(\mu)$ of solubilizates in each phase (W and A) is expressed as follows at temperature $T$ and pressure $P$ :

$$
\begin{aligned}
& \mu_{\mathrm{R}}^{\mathrm{W}}=\mu_{\mathrm{R}}^{\theta, \mathrm{W}}(T, P)+R T \ln X_{\mathrm{R}}^{\mathrm{W}} \\
& \mu_{\mathrm{R}}^{\mathrm{A}}=\mu_{\mathrm{R}}^{0, \mathrm{~A}}(T, P+\Delta P)+R T \ln X_{\mathrm{R}}^{\mathrm{A}}
\end{aligned}
$$

where $\mu^{\theta}$ is the standard chemical potential at infinite dilution $(\mathrm{W}), \mu^{0}$ is the standard chemical potential (A) based on a symmetrically standardized state, and $\Delta P$ is the difference in pressure between the $\mathrm{W}$ and $\mathrm{A}$ phases. Under the solubilization equilibrium, the following equation is obtained from Eqs. 3 and 4.

$$
\Delta G^{0}=R T \ln \frac{X_{\mathrm{R}}^{\mathrm{W}}}{X_{\mathrm{R}}^{\mathrm{A}}}
$$

$\Delta G^{0}$ means the Gibbs energy change for transfer of the solubilizate molecules from phase $\mathrm{W}$ to phase A. This is calculated according to the partition equilibrium, where the aggregate including the solubilizates is regarded as a separate phase ${ }^{12,18,20)}$. The changes of enthalpy $\left(\Delta H^{0}\right)$ and entropy $\left(\Delta S^{0}\right)$ for the transfer can be evaluated by the following equations.

$$
\begin{aligned}
& \Delta H^{0}=\left[\frac{\partial\left(\Delta G^{0} / T\right)}{\partial(1 / T)}\right]_{P} \\
& -T \Delta S^{0}=\Delta G^{0}-\Delta H^{0}
\end{aligned}
$$

The variation of $\Delta G^{0} / T$ with respect to $1 / T$ is fitted by linear regression to provide the $\Delta H^{0}$ value. The thermodynamic parameters of $\Delta G^{0}, \Delta H^{0}$, and $-T \Delta S^{0}$ are listed in Table 1 and Tables S1-3. The $\Delta G^{0}$ values of the solubilizates increase with increasing temperature (Tables S1-3). In addition, it was found that the $-T \Delta S^{0}$ term contributes more greatly to the negative value of $\Delta G^{0}$ in the whole system. Thus, the solubilization of the steroids used here to the surfactant micelle is entropy-driven under the basis of 
Table 2 Solubilization power for the $14-6-14,2 \mathrm{Br}^{-} /$ steroids/water systems.

\begin{tabular}{cccc}
\hline & PROG & $17-$ OHPC & MP \\
\hline $288.2 \mathrm{~K}$ & 0.119 & 0.0928 & 0.0904 \\
$298.8 \mathrm{~K}$ & 0.140 & 0.109 & 0.0967 \\
$308.2 \mathrm{~K}$ & 0.152 & 0.115 & 0.109 \\
\hline
\end{tabular}

release of the water molecule surrounding the steroids. As seen in Table 1, PROG and MP indicate similar $\Delta G^{0}$ values, whereas 17-OHPC has a lower value. This may be caused by a hydrophobic interaction between the hydrocarbon chains of caproic acid and $14-6-14,2 \mathrm{Br}^{-}$micelles, which is supported by a small and positive $\Delta H^{0}$ value.

\subsection{Solubilization efficacy}

The increase in solubility of poorly water-soluble compounds depends strongly on the hydrophobic room in the aqueous medium. In the case of the use of surfactants, the room is constructed by micelle formation. Thus, low cmc is a critical factor for efficacy of solubilization. Herein, we evaluated the efficacy using the solubilization power (SP) at the maximum additive concentration, which is defined as follows ${ }^{21}$ :

$$
\mathrm{SP}=\frac{\mathrm{d}[\mathrm{R}]_{\mathrm{t}}}{\mathrm{d}(C-\mathrm{cmc})}
$$

The SP index means the number of dissolved molecules in the solvent per surfactant molecule comprised in micelles. All of the SP indices increase with increase in temperature (see Table 2). At the same temperature, the solubilization efficacy of PROG in 14-6-14,2Br ${ }^{-}$solution is highest among the present steroids. This trend is different from that of the aforementioned hydrophobicity strength (17-OHPC $>$ PROG $>M P$ ). Considering the thermodynamic parameters, 17-OHPC should transfer most easily to the interior of micelles. Thus, it is likely that there are other factors of the transfer, such as interaction between the polar region of micelles and the hydrophilic groups of steroids and spatial bulkiness of steroids.

The SP value for the dodecyldimethylammonium chloride (DDAC)/sudan red B/water system at $298.2 \mathrm{~K}$ was reported to be $2.20 \times 10^{-321}$. Although DDAC is not simply the corresponding monomer of $14-6-14,2 \mathrm{Br}^{-}$gemini surfactant, it was found that the gemini surfactant can solubilize progesterone approximately 50 times more efficiently than the monomer surfactant. In a previous study, the SP index was evaluated for $14-6-14,2 \mathrm{Br}^{-} / n$-alkylbenzene/ water systems: 1.00 for ethylbenzene, 0.76 for propylbenzene, 0.30 for butylbenzene, and 0.25 for pentylbenzene at $298.2 \mathrm{~K}^{11}$. The present systems provide lower SP values. This may be caused by the hydrophilic groups of the steroids, such as the hydroxy and carbonyl groups. In the case of $n$-alkylbenzenes, elongation of the alkyl chain length

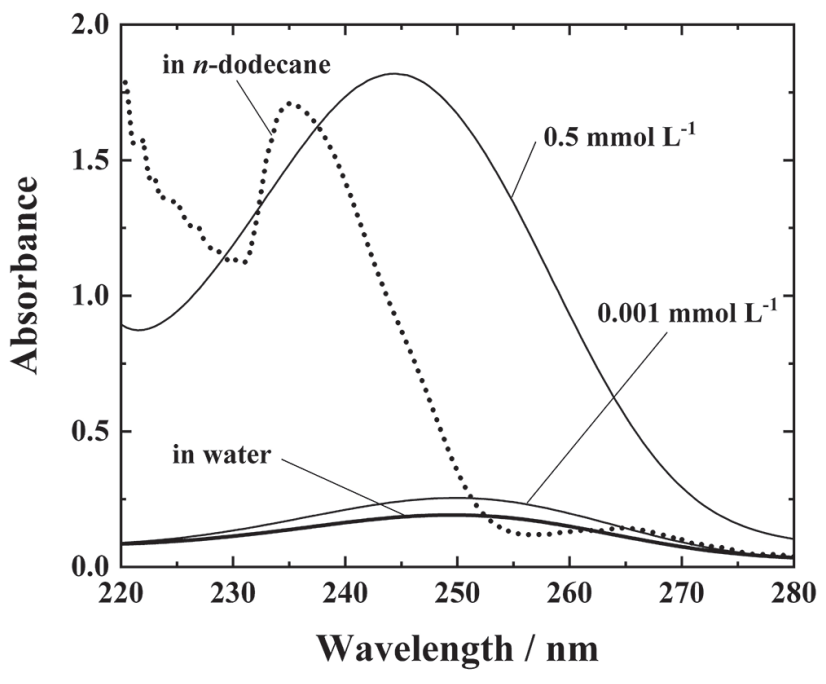

Fig. 4 Absorption spectra of PROG in water, in 14-6$14,2 \mathrm{Br}^{-}$aqueous solutions below the cmc $(0.001$ $\left.\mathrm{mmol} \mathrm{L}{ }^{-1}\right)$ and above the $\mathrm{cmc}\left(0.5 \mathrm{mmol} \mathrm{L}^{-1}\right)$, and in $n$-dodecane at $298.2 \mathrm{~K}$. The concentrations of PROG were saturated in each solvent or solution.

results in promotion of transfer to the micelle core but in degeneration of solubilization efficacy due to the limited space of hydrophobic micelle core. On the contrary, the steroids are considered to be more difficult to move into the micelle interior because of the hydrophilic groups and the bulkiness of steroid backbones.

\subsection{UV absorption spectra}

Absorption spectra of PROG in water, $14-6-14,2 \mathrm{Br}^{-}$solutions, and $n$-dodecane at $298.2 \mathrm{~K}$ are shown in Fig. 4 . The spectra of PROG in water and $0.001 \mathrm{mmol} \mathrm{L}^{-1} 14-6-14,2 \mathrm{Br}^{-}$ solution, where micelles are not formed, have a peak centered at $249.5 \mathrm{~nm}$. On the other hand, the distinct peak of the $0.5 \mathrm{mmol} \mathrm{L}^{-1}$ solution (above the cmc) appears at 244.5 $\mathrm{nm}$. In $n$-dodecane, the wavelength of the peak is exactly $235 \mathrm{~nm}$. The peak wavelength in the absorption spectrum of a molecule depends greatly on the dielectric constant of the environment in which the molecule is located. The dielectric constant for water is 78.3 and that for for $n$-dodecane is 2.0 at $298.2 \mathrm{~K}^{22)}$. Therefore, the shift of spectra reveals that most of the PROG molecules move from the aqueous bulk to the inner part of surfactant micelles. However, the peak center in $0.5 \mathrm{mmol} \mathrm{L}^{-1}$ solution did not reach $235 \mathrm{~nm}$. The spectra of $n$-alkylbenzenes in 14-6$14,2 \mathrm{Br}^{-}$solutions above the cmc nearly coincide with those in $n$-dodecane in terms of wavelength ${ }^{11)}$. Therefore, it is suggested that the solubilized PROG molecule concentrates in the micellar polar region rather than the core. 


\section{Conclusion}

The aqueous solubility of the sex hormones PROG, 17OHPC, and MP was elucidated in a micellar solution of gemini surfactant. In the presence of the micelles in the aqueous medium, it is possible for the steroids to dissolve to a greater extent in comparison with the solubility in water and the solution below the cmc. The transfer of solubilizates from the aqueous bulk to the inner part of micelles is entropy-driven for all of the systems. The UV spectra suggest the possibility that the sterols concentrate at the polar region of surfactant micelles due to the hydrophilic groups in steroids and the spatial bulkiness of the skeleton. Determining the location will necessitate further work employing other spectroscopies as well as NMR.

\section{Acknowledgements}

This work was supported by JSPS KAKENHI Grant Number 19K07036.

\section{Supporting Information}

This material is available free of charge via the Internet at http://dx.doi.org/jos.69.10.5650/jos.ess20007

\section{References}

1) Rankovic, Z. CNS drug design: Balancing physicochemical properties for optimal brain exposure. $J$. Med. Chem. 58, 2584-2608(2015).

2) Al-Safi, Z. A.; Santoro, N. Menopausal hormone therapy and menopausal symptoms. Fertil. Steril. 101, 905-915 (2014).

3) Ruan, X.; Mueck, A.O. Systemic progesterone therapyOral, vaginal, injections and even transdermal? $M a-$ turitas 79, 248-255 (2014).

4) Yalkowsky, S.H.; He, Y.; Jain, P. Handbook of Aqueous Solubility Data Second Edition. CRC Press, Boca Raton, FL (2010).

5) Biruss, B.; Valenta, C. Skin permeation of different steroid hormones from polymeric coated liposomal formulations. Eur. J. Pharm. Biopharm. 62, 210-219 (2006).

6) Vaugelade, C.; Rohmer, A.C.; Burel, F.; Belleney, J.; Duclos, R.; Bunel, C. Progesterone freeze-dried systems in sublingual dosage form. Int. J. Pharm. 229, 67-73(2001).

7) Miller, J.M.; Beig, A.; Krieg, B.J.; Carr, R.A.; Borchardt, T.B.; Amidon, G.E.; Amidon, G.L.; Dahan, A. The solubility-Permeability interplay: Mechanistic modeling and predictive application of the impact of micellar solubilization on intestinal permeation. Mol. Pharm. 8, 1848-1856 (2011).

8) Hammad, M.A.; Müller, B.W. Increasing drug solubility by means of bile salt-phosphatidylcholine-based mixed micelles. Eur. J. Pharm. Biopharm. 46, 361367 (1998).

9) Zughaid, H.; Forbes, B.; Martin, G.P.; Patel, N. Bile salt composition is secondary to bile salt concentration in determining hydrocortisone and progesterone solubility in intestinal mimetic fluids. Int. J. Pharm. 422, 295-301 (2012).

10) Barry, B.W.; Eini, D.I.D.E. Solubilization of hydrocortisone, dexamethasone, testosterone and progesterone by long-chain polyoxyethylene surfactants. J. Pharm. Pharmacol. 28, 210-218(1976).

11) Nakahara, H.; Nishizaka, H.; Iwasaki, K.; Otsuji, Y.; Sato, M.; Matsuoka, K.; Shibata, O. Role of the spacer of gemini surfactants in solubilization into their micelles. J. Mol. Liq. 244, 499-505 (2017).

12) Nakahara, H.; Kojima, Y.; Moroi, Y.; Shibata, O. Solubilization of $n$-alkylbenzenes into gemini surfactant micelles in aqueous medium. Langmuir 30, 5771-5779 (2014).

13) Zana, R. Dimeric and oligomeric surfactants. Behavior at interfaces and in aqueous solution: a review. Adv. Colloid Interface Sci. 97, 205-253(2002).

14) Menger, F.M.; Keiper, J.S. Gemini surfactants. Angew. Chem. Int. Ed. 39, 1906-1920 (2000).

15) Zana, R.; Benrraou, M.; Rueff, R. Alkanediyl- $\alpha, \omega$-bis (dimethylalkylammonium bromide) surfactants. 1. Effect of the spacer chain length on the critical micelle concentration and micelle ionization degree. Langmuir 7, 1072-1075(1991).

16) Nakahara, H.; Nishino, A.; Tanaka, A.; Fujita, Y.; Shibata, O. Interfacial behavior of gemini surfactants with different spacer lengths in aqueous medium. Colloid Polym. Sci. 297, 183-189(2019).

17) Schwarz, D.H.; Engelke, A.; Wenz, G. Solubilizing steroidal drugs by $\beta$-cyclodextrin derivatives. Int. J. Pharm. 531, 559-567(2017).

18) Sato, Y.; Nakahara, H.; Moroi, Y.; Shibata, O. Solubilization of $n$-alkylbenzenes into octaethylene glycol mono$n$-tetradecyl ether $\left(\mathrm{C}_{14} \mathrm{E}_{8}\right)$ micelles. Langmuir 23, 7505-7509 (2007).

19) Matsuoka, K.; Hirosawa, T.; Honda, C.; Endo, K.; Moroi, Y.; Shibata, O. Thermodynamic study on competitive solubilization of cholesterol and $\beta$-sitosterol in bile salt micelles. Chem. Phys. Lipids 148, 51-60 (2007).

20) Take'uchi, M.; Moroi, Y. Solubilization of $n$-alkylbenzenes into 1-dodecanesulfonic acid micelles. Langmuir 11, 4719-4723(1995).

21) Ozeki, S.; Ikeda, S. The difference in solubilization power between spherical and rodlike micelles of dodecyldimethylammonium chloride in aqueous solu- 
H. Ideguchi, Y. Takasaki, S. Kondo et al.

tions. J. Phys. Chem. 89, 5088-5093(1985).

22) Haynes, W.M. CRC Handbook of Chemistry and
Physics. 91st ed. CRC Press, Boca Raton, London (2010). 